\title{
ESPECIES DE TRIPS (INSECTA: THYSANOPTERA: THRIPIDAE) EN LAS FLORES DE ORNAMENTALES
}

\author{
TRIPS SPECIES (INSECTA: THYSANOPTERA : THRIPIDAE) \\ IN THE ORNAMENTAL FLOWERS (CROPS).
}

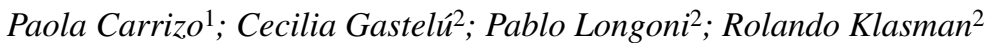

\begin{abstract}
RESUMEN
Los trips (Thysanoptera: Thripidae) son polífagos y, por lo tanto, son capturados en muchas especies de plantas cultivadas, lo cual no implica que en ellas se comporten como plagas. El objetivo de este trabajo fue relevar la presencia y abundancia trips, en los cultivos de especies ornamentales. En febrero de 2005 se colectaron flores de 65 especies de plantas, hallándose individuos adultos en diversos niveles de densidad en 49 de ellas. Las especies de trips más frecuentes y abundantes fueron: Thrips simplex (Morison) (trips del gladiolo), Frankliniella schultzei Trybom (trips de los capullos), Frankliniella occidentalis (Pergande) (trips californiano de las flores), Frankliniella insularis Franklin (trips del malvabiscus) y Frankliniella gemina Bagnall. Para T. simplex, plaga del gladiolo, se registró en sus flores una abundancia media (por flor) de 5 a 10 individuos (considerando adultos, juveniles o ambos). Niveles similares de densidad se registraron en otras plantas: F. schultzei en Lathyrus latifolius, Ipomea y Tropaleum majus, F. insularis en Punica granatum, Malvaviscus arboreus, Hibiscus rosa sinensis y Duranta erecta, y F. gemina en Agapanthus africanus y Dietes bicolor. Se espera que resultados preliminares puedan orientar futuras investigaciones sobre la interacción de especies de trips con estos cultivos.
\end{abstract}

Palabras clave: trips, plantas ornamentales, plagas.

\begin{abstract}
Since thrips (Thysanoptera: Thripidae) are polyphagous, they are usually caught in flowers of many crops, but it does not necessary mean they are pests. The aim of this survey was to list the thrips presence and abundance in ornamental crops. During february 2005 were collected flowers form 65 plant species, and they were recorded thrips, in different abundance levels in 49 plant species. The thrips species more frequently found were Thrips simplex (Morison) (gladiolus thrips), Frankliniella schultzei Trybom (blossom thrips), Frankliniella occidentalis (Pergande) (western flower thrips), Frankliniella insularis Franklin (malvaviscus thrips) and Frankliniella gemina Bagnall. For T. simplex - a gladiolus pest - it was recorded a mean abundance of 5 to 10 individuals per flower in that crop (considering larvae, adults or both). Similar densities were recorded also for F. schultzei in Lathyrus latifolius, Ipomea and Tropaleum majus, F. insularis in Punica granatum, Malvaviscus arboreus, Hibiscus rosa sinensis and Duranta erecta, and for $\mathrm{F}$. gemina in Agapanthus africanus and Dietes bicolor. It is expected these preliminary results may assist in the establishment of priorities for future research on thrips-crops interaction.
\end{abstract}

Key words: thrips, ornamental crops, pests.

\section{INTRODUCCIÓN}

La mayor parte de los trips (Thripidae) presentan una amplia flexibilidad alimentaria y requiere de polen para completar su desarrollo y asegurar su fertilidad (Mound y Marullo, 1996). Como consecuencia, ha sido reportada la captura de varias especies de trips en las flores de una considerable lista de plantas, varias de ellas de valor ornamental; sin embargo, muchas de estas capturas podrían ser

1 Zoología Agrícola. Facultad de Agronomía. Universidad de Buenos Aires. Av. San Martín 4453. Ciudad Autónoma de Buenos Aires. C1417DSE. Argentina

2 Floricultura. Facultad de Agronomía. Universidad de Buenos Aires. Av. San Martín 4453. Ciudad Autónoma de Buenos Aires. C1417DSE. Argentina. E-mail: pcarrizo@agro.uba.ar 
casuales. Por otra parte, la presencia de un artrópodo fitófago en un cultivo no implica que le ocasione daños desde el punto de vista económico. A fin de ayudar a establecer objetivamente prioridades de investigación para los trips plagas, se relevó la presencia y abundancia de trips en especies ornamentales.

\section{MATERIALES Y MÉTODOS}

Durante el mes de febrero de 2005 se colectaron flores de las plantas cultivadas (Brickell et $a l ., 2004)$ en el jardín de la escuela. Las muestras $(\mathrm{n}=5$ /especie de planta) fueron colocadas en bolsas plásticas y preservadas en heladera hasta su recuento. Los ejemplares de trips presentes fueron contados, registrándose la presencia de formas inmaduras y adultos, identificándose estos últimos mediante claves sistemáticas (De Santis, et al., 1980; Moritz et al., 2001; Mound \& Marullo, 1996; Nakahara, 1994).

\section{RESULTADOS Y DISCUSIÓN}

En la siguiente Tabla 1 se detalló la abundancia y presencia de trips, en aquellas especies de plantas en las cuales se hallaron ejemplares vivos. En la mayoría de los casos se registró la presencia de adultos en diferentes niveles de abundancia; la ocurrencia de inmaduros fue ocasional y en muy baja abundancia, correspondiendo a Thripinae (Speyer y Parr, 1941). Esto coincide con la observación de que los adultos de una especie pueden hallarse en cientos de plantas, mientras que las larvas pueden ser halladas en relativamente pocas (Carrizo, 2002, Kirk, 1985).

Respecto de los trips, Mound y Marullo (1996) puntualizan que una planta puede considerarse como un hospedante, si ha sido registrada repetidamente la presencia de una especie de trips determinada, junto con sus inmaduros. En los Thripinae polenófagos los adultos se agrupan por miles en las flores abiertas y las utilizan como sustrato para la cópula, aunque no dejen en ellas a su descendencia (Kirk, 1985).

En un cultivo ornamental, su aspecto es decisivo y los daños que los trips producen con su aparato bucal deprecian el producto y lo inutilizan para su comercialización, aun cuando no dejen descenden- cia en la misma planta. De tal modo, el impacto que provocan se relaciona exclusivamente con su abundancia (Kirk, 1997), independientemente de la posibilidad de producir inmaduros al alimentarse de esa planta.

¿A partir de qué densidad es dable considerar a un artrópodo como plaga, ya que produce daños al cultivo? El gladiolo es una hospedante verdadera para $T$. simplex, ya que en esta planta completa su ciclo y produce descendencia; asimismo, en ella se comporta como plaga (de Santis et al., 1980). En los relevamientos presentados, se tomaron muestras de Gladiolus, en cuyas flores se hallaron densidades de alrededor de 5 a 10 individuos/flor, dependiendo si se consideran las formas juveniles o adultas, separadas o conjuntamente. Tomando este valor como referencia, al leer la Tabla 1 pueden hallarse especies de plantas en las cuales esta densidad fue alcanzada, e incluso superada. Asimismo, cuando los trips se encontraron en estas densidades, existió una clara dominancia por una especie, excepcionalmente dos.

Frankliniella gemina (endémica sudamericana) fue hallada en alta densidad y dominó la captura en: Agapanthus, Clivia, Dhalia, Lagerstroemia, Lantana, Pandorea y Rosa. El trips del malvavisco, Franliniella insularis, fue la especie dominante en Duranta, Hibiscus, Malvaviscus y Punica, mientras que Frankliniella schultzei (el trips de los capullos) hizo lo propio en Lathyrus y Tropaelum y, por su parte, T. simplex (el trips del gladiolo) presentó altas capturas sólo en Gladiolus.

El trips de las compuestas, Microcephalothrips abdominalis, se halló en altas densidades y dominó en Coreopsis y Gazania. Frankliniella occidentalis (el trips californiano de las flores), uno de los Thripinae característico por su polifagia y plaga de invernadero, sólo presentó alta densidad en Gypsophylla y el segundo lugar en dominancia -luego de F. gemina- en Polianthes.

Debe destacarse que en varios casos en los cuales no se alcanza esta densidad mínima de 5 individuos/ flor (Abelia, Abutilon, Campsis, Ceratostigma, Cosmos, Crinun, Eichornia, Impatiens o Nerium, Passiflora y Polygala) también una especie de trips dominó la captura.

Los recursos disponibles para la investigación son en general limitados y se hace necesario priorizar su destino hacia las especies que están actuando como plagas, esto es, que puedan 
producir daños apreciables desde el punto de vista económico. Se espera que el relevamiento presentado actúe como una contribución al res- pecto, dada la escasez de información para los trips en particular y para aquellos endémicos y/o en cultivos ornamentales.

Tabla 1

Abundancia e identidad de Thysanoptera presentes en las flores de las plantas relevadas

\begin{tabular}{|c|c|c|c|c|c|c|c|c|c|c|c|}
\hline & & Tot & $\mathbf{I}$ & Fo & Fs & $\mathbf{F i}$ & Ch & Ts & $\mathbf{H}$ & Ma & Fg \\
\hline 1 & Abelia grandiflora & 11 & 2 & & 1 & & & & & & 8 \\
\hline 2 & Abutilon sp. & 4 & & & & 4 & & & & & \\
\hline 3 & Agapanthus africanus & 31 & & & 1 & 1 & & & & & 29 \\
\hline 4 & Alstroemeria híbrida IRENA & 3 & & 3 & & & & & & & \\
\hline 5 & Alstroemeria psittacina & 7 & & & 1 & 4 & & & & & 2 \\
\hline 6 & Bougainvillea spectabilis & 8 & 1 & & & & & & & & 7 \\
\hline 7 & Bulbine caulescens & 7 & & & 4 & 1 & & & & & 2 \\
\hline 8 & Callistemon lanceolatus & 8 & 2 & & & 2 & & & & & 4 \\
\hline 9 & Campsis radicans & 16 & 3 & & & 11 & & & & & 2 \\
\hline 10 & Canna sp. & 3 & & & 2 & 1 & & & & & \\
\hline 11 & Ceratostigma plumbaginoides & 13 & & 2 & 7 & & & & 2 & & 2 \\
\hline 12 & Clivia rucinata & 24 & & & & & & & & & 24 \\
\hline 13 & Coreopsis grandiflora & 43 & & & & 1 & & & & 41 & 1 \\
\hline 14 & Cosmos sp. & 7 & & & & & & & & 7 & \\
\hline 15 & Crinum sp. & 14 & 1 & 1 & 1 & 1 & & & & & 10 \\
\hline 16 & Dhalia sp. & 25 & & & & 2 & & & & & 23 \\
\hline 17 & Dietes bicolor & 8 & & 1 & & & & & & & 7 \\
\hline 18 & Duranta erecta & 35 & & & & 25 & & & & & 10 \\
\hline 19 & Eichornia crassipes & 9 & & & & 9 & & & & & \\
\hline 20 & Eustoma sp. & 2 & & & & & & & & & 2 \\
\hline 21 & Fuchsia magallanica & 2 & & & & 2 & & & & & \\
\hline 22 & Gazania splendens & 28 & & 4 & 3 & & & & & 18 & 3 \\
\hline 23 & Gladiolus sp. & 50 & 23 & & & & & 27 & & & \\
\hline 24 & Gypsophylla paniculata & 51 & 37 & 10 & 4 & & & & & & \\
\hline 25 & Hibiscus rosa sinensis & 107 & 3 & & & 104 & & & & & \\
\hline 26 & Impatiens wallerana (fucsia) & 19 & & & & 18 & & & & & 1 \\
\hline 27 & Impatiens wallerana (rosa) & 1 & & & & & & & & & 1 \\
\hline 28 & Ipomea sp. & 22 & & & 21 & & & & & & 1 \\
\hline 29 & Lagerstroemia indica & 27 & 1 & 1 & & & & & 1 & & 24 \\
\hline 30 & Lantana camara (amarilla) & 22 & 1 & & 3 & 2 & & & & & 16 \\
\hline 31 & Lantana camara-(blanca) & 30 & & & 4 & & & & & & 26 \\
\hline
\end{tabular}




\begin{tabular}{|c|c|c|c|c|c|c|c|c|c|c|c|}
\hline & & Tot & $\mathbf{I}$ & Fo & Fs & $\mathbf{F i}$ & Ch & Ts & $\mathbf{H}$ & Ma & Fg \\
\hline 32 & Lathyrus latifolius & 47 & & 5 & 27 & 10 & & & & & 5 \\
\hline 33 & Limonium sinuatum & 5 & & & & 1 & & & 1 & & 3 \\
\hline 34 & Malvaviscus arboreus & 93 & & & & 91 & & & & & 2 \\
\hline 35 & Nerium oleander & 13 & & & & 1 & & & & & 12 \\
\hline 36 & Pandorea-jasminoides & 26 & 1 & & & 11 & & & & & 14 \\
\hline 37 & Passiflora coerulea & 7 & & & & & & & & & 7 \\
\hline 38 & Polianthes tuberosa & 34 & & 10 & & & & & & & 24 \\
\hline 39 & Polygala myrtifolia & 19 & & 1 & 4 & 1 & 1 & & & & 12 \\
\hline 40 & Punica granatum & 63 & & 1 & & 56 & & & & & 6 \\
\hline 41 & Rosa $x$ hybrida (blanca) & 12 & & 2 & 3 & 3 & 2 & & & & 2 \\
\hline 42 & Rosa x hybrida (de té) & 55 & & & 1 & 6 & & & & & 48 \\
\hline 43 & Rosa $x$ hybrida (roja) & 9 & & 1 & 1 & 6 & & & & & 1 \\
\hline 44 & Rudbeckia sp. & 8 & & & & 1 & & & & 6 & 1 \\
\hline 45 & Salvia farinacea & 5 & & 1 & 1 & 1 & & & & & 1 \\
\hline 46 & Salvia splendens & 2 & & & 2 & & & & & & \\
\hline 47 & Tropeaelum majus & 25 & & 1 & 24 & & & & & & \\
\hline 48 & Thubalghia violacea & 18 & 7 & 6 & 3 & & & & & & 4 \\
\hline 49 & Zantedeschia heliothiana & 2 & & 2 & & & & & & & 2 \\
\hline
\end{tabular}

Referencias:

Tot.: total de ejemplares en la muestra (en 5 flores)

T.s. : Trips simplex (Morison)

H. : Haplothrips Bournier

F.o. : Frankliniella occidentalis (Pergande)

F.i. : Frankliniella insularis (Franklin)

\section{LITERATURA CITADA}

BRICKELL, C.; BAUM, B.; HETTERSCHEID, W.; LESLIE, A.; MCNEILL, J.; TREHANE, P.; VRUGTMAN, F.; WIERSEMA, J. 2004. International Code of Nomenclature for cultivated plants. 7th Edition. IUBS Commission for the Nomenclature of Cultivated Plants. Regnum Vegetabile, 144.

CARRIZO P. 2002. Establishing a weed host ranking for thrips vectors of tospovirus in La Plata horticultural belt (Buenos Aires, Argentina). pp 239-248. In: Section VII: Control strategies. Proceedings, Fifth International Symposium of Thrips and Tospoviruses, Reggio, Calabria, Italy. Marullo, R and L. Mound (eds.) ISBN 0-9750206 0-9. Dec. 2002.

DE SANTIS, L.; GALLEGO DE SUREDA, A.E.; MERLO, E.Z. 1980. Estudio sinóptico de los tisanópteros argentinos. (Insecta). Obra del Centenario Museo LP, VI: 91-166.

KIRK,-W.D.J. 1985. Aggregation and mating of thrips in flowers of Calystegia sepium. Ecological entomology 10: 433-440.

KIRK, W.D.J. 1997. Feeding. Cap. 4.: 119-174. In: Thrips as crop pests. Ed. T. Lewis. CAB International.
I : inmaduros

Ch. : Chirothrips (Morison)

F.s. : Frankliniella schultzei Trybom

F.g. : Frankliniella gemina Bagnall

M.a. : Microcephalothrips abdominalis Crawford
LA ROSSA, R.; BADO, S.; PANNUNZIO, M.J. 2004. Nota sobre la presencia de Frankliniella insularis (Thysanoptera: Thripidae) en la República Argentina. Revista de la Facultad de Agronomía 24 (2): 113-117.

MORITZ, G.; MORRIS, D.; MOUND, L. 2001. Thrips ID. Pest thrips of the world. An identification and information systems. CSIRO Publications. Australia.

MOUND, L.A.; MARULLO, R. 1996. The thrips of Central and South America. An Introducion (Insecta: Thysanoptera). Memoirs on Entomol., Int. V 6. Assoc. Publ. Fla., USA. $487 \mathrm{pp}$.

NAKAHARA, S. 1994. The genus Thrips Linnaeus (Thysanoptera: Thripidae) of the New World. USDA Technical Bulletin 1822. $183 \mathrm{pp}$.

SPEYER, E.R.; PARR, W.J. 1941. The external structure of some Thysanopterous larvae. Trans. R. Entomol. Soc. Lond. 91: 559-653. 

ANDROS IMPRESORES

www.androsimpresores.cl 